\title{
Automorphism Groups of Models of First order Theories.
}

\author{
V. V. Bludov ${ }^{\dagger}$, M. Giraudet, A. M. W. Glass and G. Sabbagh \\ 19th January 2007
}

To A. L. S. Corner, in memoriam.

\begin{abstract}
Some years ago (see [5], Problem 12.14), we asked if every first order theory having infinite models has a model whose automorphism group has undecidable theory. In this note, we prove that this is true in a strong form. It will be a consequence of our other theorem that there is a right orderable finitely presented group with insoluble word problem.
\end{abstract}

$\dagger$ Vasily Bludov was supported by the Russian Foundation for Basic Research, grant no. 07-01-00596.

AMS Classification: 03B25, 06F15, 20B27.

Keywords: finitely presented group, insoluble word problem, right ordered group, automorphism group, undecidable theory, existential theory. 


\section{Introduction}

Recall that a group $G$ is right orderable if there is a total order $\leq$ on $G$ such that $f \leq g$ in $G$ implies that $f h \leq g h$ for all $h \in G$.

We will prove

Theorem A There is a right orderable finitely presented group with insoluble word problem.

and deduce

Theorem B Every first order theory having infinite models has a model whose automorphism group has undecidable existential theory. Moreover, such models exist in all infinite cardinals which are at least the cardinality of the language.

\section{Proof that Theorem A implies B.}

As noted in [6], Example 1.2.3, if $G$ is any right ordered group, then $G$ can be embedded (as a group) in $\operatorname{Aut}(G, \leq)$. But if $(\Omega, \leq)$ is any countable totally ordered set, then the group $\operatorname{Aut}(\Omega, \leq)$ can be embedded in $\operatorname{Aut}(\mathbb{Q}, \leq)$, where $(\mathbb{Q}, \leq)$ is the set of rational numbers with the usual standard ordering (see [3], Theorem 2.J). Hence we obtain the following (folklore):

Lemma 2.1 Every countable right orderable group can be embedded in $\operatorname{Aut}(\mathbb{Q}, \leq)$.

We now use this to deduce Theorem B from Theorem A.

Proof: Let $G$ be any right orderable finitely presented group with insoluble word problem; say,

$$
G:=\left\langle x_{1}, \ldots, x_{m}: u_{1}\left(x_{1}, \ldots, x_{m}\right)=1, \ldots, u_{n}\left(x_{1}, \ldots, x_{m}\right)=1\right\rangle .
$$

For any word $w\left(x_{1}, \ldots, x_{m}\right)$, let $\psi_{w}$ be the sentence

$$
\forall x_{1}, \ldots, x_{m}\left[\left(\bigwedge_{i=1}^{n} u_{i}\left(x_{1}, \ldots, x_{m}\right)=1\right) \rightarrow w\left(x_{1}, \ldots, x_{m}\right)=1\right] .
$$

Let $H$ be any group containing $G$. If $H \models \psi_{w}$, then $G \models \psi_{w}$ since $\psi_{w}$ is a universal sentence ([2], Theorem 5.2.4). But each $u_{j}\left(x_{1}, \ldots, x_{m}\right)=1$ in $G$

$(j=1, \ldots, n)$, so $w\left(x_{1}, \ldots, x_{m}\right)=1$ in $G$ if $H \models \psi_{w}$. Conversely, suppose 
that $w\left(x_{1}, \ldots, x_{m}\right)=1$ in $G$. Let $h_{1}, \ldots, h_{m} \in H$ with $u_{j}\left(h_{1}, \ldots, h_{m}\right)=1$ in $H(j=1, \ldots, n)$. Then the subgroup of $H$ generated by $h_{1}, \ldots, h_{m}$ is a homomorphic image of $G$. Hence $w\left(h_{1}, \ldots, h_{m}\right)=1$ in $H$. Thus $H \models \psi_{w}$. So $w\left(x_{1}, \ldots, x_{m}\right)=1$ in $G$ if and only if $H \models \psi_{w}$. Since the word problem for $G$ is insoluble, the universal theory of $H$ is undecidable. Therefore the existential theory of $H$ must also be undecidable. By Lemma 2.1, we may take $H$ to be $\operatorname{Aut}(\mathbb{Q}, \leq)$; so the latter has undecidable existential theory. But if $T$ is any first order theory having infinite models, then it has a model $\mathcal{M}$ built using Skolem functions with indiscernibles $(\mathbb{Q}, \leq)$; moreover, every element of $\operatorname{Aut}(\mathbb{Q}, \leq)$ extends uniquely to an element of $A u t(\mathcal{M})$ (see, [2], Section 3.3). We therefore have embeddings of $G$ into $\operatorname{Aut}(\mathbb{Q}, \leq)$ and $\operatorname{Aut}(\mathbb{Q}, \leq)$ into $A u t(\mathcal{M})$. By letting $H$ above be $\operatorname{Aut}(\mathcal{M})$, we see that $\operatorname{Aut}(\mathcal{M})$ has undecidable existential theory. Consequently, $\mathcal{M}$ is the desired the model of $T$ and the first part of Theorem B follows.

For the general case, let $\mathcal{L}$ be the language of $T$ and $\kappa \geq\|\mathcal{L}\|$ be an infinite cardinal thought of as an ordinal. Let $(X, \leq)$ be the disjoint union of $\mathbb{Q}$ and $\kappa$, totally ordered by letting every rational number be less than every element of $\kappa$. Build a model $\mathcal{M}$ of $T$ using Skolem functions and indiscernibles $(X, \leq)$. It has cardinality $\kappa$. Now $\operatorname{Aut}(\mathbb{Q}, \leq)=\operatorname{Aut}(X, \leq)$ which can be embedded in $\operatorname{Aut}(\mathcal{M})$. By the same proof as in the previous paragraph, $\operatorname{Aut}(\mathcal{M})$ has undecidable existential theory. This completes the deduction of Theorem B from Theorem A. //

\section{Proof of Theorem A.}

Let $m$ be a positive integer with $m \geq 2$, and $F_{m}$ be the free group on $m$ free generators. Let $P:=F_{m} \times F_{m}$. Then there is a finitely generated subgroup $H$ of $P$ such that the membership problem for $H$ (in $P$ ) is insoluble ([7], Theorem 4.3). That is, there is no algorithm which determines for an arbitrary element of $P$ whether it belongs to $H$ or not. Let $H$ be generated by $h_{1}, \ldots, h_{n}$, say. Consider the group

$$
G:=\left\langle P, t:\left[t, h_{i}\right]=1(i=1, \ldots, n)\right\rangle .
$$

We establish Theorem A by proving

Proposition 3.1 G is a right orderable finitely presented group with insoluble word problem.

Proof: The proof relies on a result of George Bergman ([1], Theorem 35): 
Let $\left\{G_{i}: \quad i \in I\right\}$ be a family of right orderable groups having a common subgroup $S$. Suppose that there is a group $U$ and embeddings $\varphi_{i}: G_{i} \rightarrow U$ with $s \varphi_{i}=s \varphi_{j}$ (for all $s \in S$ and $\left.i, j \in I\right)$. Then the free product of $\left\{G_{i}: i \in I\right\}$ with $S$ amalgamated is right orderable.

$P$ can be given by the finite presentation

$$
P=\left\langle a_{1}, \ldots, a_{m}, b_{1}, \ldots, b_{m}:\left[a_{i}, b_{j}\right]=1(i, j \in\{1, \ldots, m\})\right\rangle .
$$

Hence $G$ is finitely presented. Next, $G$ has insoluble word problem, since otherwise we could solve the membership problem for $H$ in $P$ (an element $c \in P$ belongs to $H$ iff $[t, c]=1$ in $G-[7]$, Section IV.2). Finally, we need to show that $G$ is right orderable. Now any free group is orderable ([4], Example 1.3.24). Hence so is $P$ (use the lexicographic order). Let $N$ be the normal subgroup of $G$ generated by $P$. Then $N$ is a free product of orderable groups $\left\{t^{-k} P t^{k}: k \in \mathbb{Z}\right\}$ with the same subgroup $H$ of each amalgamated. For each $k \in \mathbb{Z}$, let $\varphi_{k}: t^{-k} P t^{k} \rightarrow P$ be the natural isomorphism given by: $t^{-k} p t^{k} \mapsto p$. By our hypotheses, all $\varphi_{k}(k \in \mathbb{Z})$ agree on $H$. By Bergman's result (with $I=\mathbb{Z}, G_{k}=t^{-k} P t^{k}, S=H$ and $U=P$ ), we get that $N$ is right orderable. Let $\leq_{N}$ be such a right order on $N$. Every element $g$ of $G$ can be written uniquely in the form $g=x t^{\ell}$ with $x \in N$ and $\ell \in \mathbb{Z}$. Let $h=y t^{\ell^{\prime}}$ with $y \in N$ and $\ell^{\prime} \in \mathbb{Z}$. Define $g \leq h$ in $G$ if and only if $\ell<\ell^{\prime}$ (in $\mathbb{Z}$ ) or both $\ell=\ell^{\prime}$ and $x \leq_{N} y$. This gives a right ordering on $G$ and establishes the theorem. //

For future reference we observe that we have proved

Corollary 3.2 If $G$ is a right orderable group with subgroup $H$, then the group $\langle G, t:[t, h]=1(h \in H)\rangle$ is right orderable.

Also, as mentioned in the proof of the deduction of Theorem B from Theorem A, we have that

Corollary 3.3 Aut $(\mathbb{Q}, \leq)$ has undecidable existential theory.

Acknowledgements: This solution to Problem 12.14 in [5] was obtained as a result of discussion at the "Colloque Giraudet" conference. We wish to thank Modnet MRTN-CT-2004-512234 for funds for this meeting and for covering the expenses of the third author. 


\section{References}

[1] G. M. Bergman, Ordering coproducts of groups and semigroups, J. Algebra 133 (1990), 313-339.

[2] C. C. Chang and H. J. Keisler, Model Theory, Studies in Logic 73, NorthHolland Publishing Co., Amsterdam, 1973.

[3] A. M. W. Glass, Ordered permutation groups, London Math. Society Lecture Notes Series 55, Cambridge University Press, Cambridge, 1981.

[4] A. M. W. Glass, Partially ordered groups, Series in Algebra 7, World Scientific Publishing Co., Singapore, 1999.

[5] Unsolved Problems in Group Theory: Kourovka Note Book, Novosibirsk.

[6] V. M. Kopytov and N. Ya. Medvedev, Right Ordered Groups, Kluwer Publishing Co., Dordrecht, 1996.

[7] R. C. Lyndon and P. E. Schupp, Combinatorial Group Theory, Ergebnisse der Math. 89, Springer-Verlag, Heidelberg, 1977.

Authors' addresses:

V. V. Bludov:

Department of Mathematics, Physics, and Informatics, Irkutsk Teachers Training University,

Irkutsk 664015,

Russia

vasily-bludov@yandex.ru

M. Giraudet

Équipe de Logique, Université Paris 7, 2 Place Jussieu, 75251 Paris cedex 05,

France

giraudet@logique.jussieu.fr 
A. M. W. Glass:

Department of Pure Mathematics and Mathematical Statistics, Centre for Mathematical Sciences,

Wilberforce Rd., Cambridge CB3 0WB,

England

amwg@dpmms.cam.ac.uk

G. Sabbagh:

Équipe Logique et département de Mathématiques, Université Paris 7,

2 Place Jussieu, 75251 Paris cedex 05,

France

sabbagh@logique.jussieu.fr 